\title{
Blockchain-based model to track and verify official certificates
}

\author{
Pooja Mara ${ }^{1}$, Ravi kanth Motupalli ${ }^{2}$ \\ ${ }^{I}$ VNR Vignana Jyothi Institute of Engineering and Technology, Hyderabad, India \\ ${ }^{2}$ VNR Vignana Jyothi Institute of Engineering and Technology, Hyderabad, India \\ ${ }^{1}$ poojamara999@gmail.com \\ 2ravikanth_mevnrvjiet.in
}

\begin{abstract}
Document verification is a complicated domain with a variety of difficult and time-consuming methods to validate. Customized verification and authentication processes may be required for various sorts of papers, such as financial papers, government papers, transaction papers, educational credentials, and so on. A huge problem today we are facing is the number of fake certificates that are in circulation, this problem is quite predominant. This has become a new business for a long time. Hardworking people with genuine degrees/certificates have been suffering and they get rejected in the job market because of the lack of identification to differentiate between the original and the fake certificate. At times people are getting jobs through fake certificates and this becomes very dangerous. The scenario calls for a new system that can verify and authenticate certificates, their issuers, and their holders in a way that is much more efficient, simple, and intuitive to use, and efficiently mitigates widespread credential fraud. To combat the counterfeiting of academic certificates, our blockchain approach combines a verified distributed ledger with a cryptographic mechanism. Our Blockchain technology will also provide a standard sharing platform for storing and accessing documents, reducing overall verification time and allowing companies to quickly monitor and access real papers. Our system that helps to identify original certificates will help the hard-earned people to get their jobs in their desired organizations and all the fake circulation of certificates will be stopped because the system identifies the genuine true certificates.
\end{abstract}

Keywords - Educational Certificates verification, authentication, Blockchain, cryptography, Web Application.

\section{INTRODUCTION}

Students who have finished the course at that level receive certificates from universities and other boards. The majority of these certifications are in the form of paper papers, and an electronic document cannot effectively replace a physical certificate. Due to the availability of sophisticated and inexpensive scanning and printing technologies, however, certificate fraud has increased. This has an impact on the owners of the certificates as well as the boards of the university that issued them. It is required to verify that the graduate's graduation certificate is legitimate, and that the holder is the legitimate owner.
Furthermore, education credentials must be validated to confirm that the content is valid and that the certificate is obtained from a reliable source. Many hours will be spent contacting the university to get a certificate approved or requesting a statement from the institution to guarantee that the certificate is proper, and that the verification is appropriate to validate its validity. This procedure can be time-consuming and costly. One advantage of digital systems is that certification may be verified instantaneously. We've decided to look into the field of Blockchain in our project to put our idea into action

Blockchain is a means of storing data in such a way that altering, hacking, or defrauding it is difficult or impossible. A Blockchain is a digital $\log$ of transactions that is duplicated and disseminated throughout the network of computer systems that make up the Blockchain. Each block on the chain contains several transactions, and whenever a new transaction occurs on the Blockchain, a record of it is recorded to each participant's ledger. Distributed Ledger Technology (DLT) is a decentralized database managed by several individuals (DLT). Blockchain is a distributed ledger system in which transactions are recorded using a hash, which is a cryptographic signature that cannot be changed. This means that if one block in a chain is changed, it will be obvious that the entire chain has been tampered with. If hackers wanted to take down a Blockchain system, they'd have to change every block in the chain across all distributed versions. As additional blocks are added to the chain, Blockchain like Bitcoin and Ethereum grow in size, greatly boosting the security of the ledger.

\section{RELATED WORK}

Paper [1] is a Blockchain concept proposed by Satoshi Nakamoto in 2008. Blockchain is an internet platform that allows for decentralized and transparent data transfer. All transaction data (stored on nodes) will be compressed and linked to distinct blocks via distributed recordings. Discrete categories of data are delivered in different blocks, allowing for verification without the involvement of intermediaries. All of the nodes join together to construct a Blockchain with timestamps that will simultaneously 


\section{International Journal of Engineering Technology and Management Sciences}

Website: ijetms.in Issue: 1 Volume No.6 January - 2022 DOI: 10.46647/ijetms.2022.v06i01.002

ISSN: $2581-4621$

validate the data in each block and become unchangeable until they are connected. The entire process is free, transparent, and safe for the public.

The operating procedures of the system built in this investigation are provided in the publication [2]. The following are some examples: A degree certificate is awarded by the school, and the student's certificate is entered. Information is accessed via the device. After then, the gadget will operate on its own. It uses a Blockchain to store the student's serial number. The certificate system verifies all of the information. Instead of traditional physical copies, schools issue E-certificates with the Fast Reply (QR) code to e-certificate Graduates whose knowledge has been satisfactorily validated. In addition, each graduate receives an inquiry number for their electronic certificate file. When applying for a job, a graduate just sends the post. Businesses can use a $\mathrm{QR}$ code to send a serial number or an e-certificate to the target. Businesses make scheme inquiries, and if the serial numbers are confirmed, they are alerted. The QR Code enables them to determine if the certificate has been tampered with or faked.

According to the report [3], all the solutions provide insufficient security in terms of covering all of the five issues highlighted in the research. As a result of the examination, it was discovered that in the suggested system solution cases the certificate is vulnerable to attack and data security is inadequate. As a result of the online solution assessments, the authentication, authorization, confidentiality, privacy, and ownership holes were discovered in existing certificate verification systems. As a result, the goal of this study was to fill in the gaps by presenting a blockchain-based architecture for academic certificate verification that focused on authentication, authorization, secrecy, privacy, and ownership. The suggested framework is based on the Hyper Ledger Fabric framework; hence the suggested system is built on it.

In paper [4], they may construct unmodifiable certificates based on Blockchains, but first, the university must register. Each university will have its wallet address to which transactions will be sent. Only the smart contract's owner can add universities. The university will have access to the system and will be able to generate certificates with data fields after it has been uploaded. Each certificate will be kept in the Interplanetary File System (IPFS), which will return the SHA-2S6 algorithm's unique hash. All of this information, including the produced hash and certificate details, will be saved in the Blockchain.

The purpose of this paper [5] is to employ Blockchain technology to overcome the difficulties with the present certificate verification system, as well as to prevent user fraud and provide transparency in the education system. The goal of this project is to see how well Blockchain can be used to construct distributed systems as a service. The administrator has the authority to affirm the administrator in the digital fields used to check a user's identity and authorization to enter the network, thanks to the digital certificate's usage of digital signature technology.

Paper [6] describes a system that uses double SHA256 to create the hash value for a certificate. In the block, save the fixed-length hash value as a transaction. The members of the Blockchain validate this transaction, and if it is deemed to be a genuine transaction, the block is added to the current Blockchain. The consensus algorithm will be used to accept and reject proposals. The number of nodes and transactions might influence the consensus method. The system will create a $\mathrm{QR}$ code and an inquiry string code to be included in the hardcopy certificate. The solution allows the unit to verify the authenticity of a hardcopy certificate using a phone scanner or a website. Because of the distributed ledger's immutability, the system not only verifies certificates but also maintains them in digital form indefinitely.

The academic and athletic certifications are turned into digital certificates utilizing sampling and quantization in this study [7]. The certificates are then combined with the digital certificate's hash values and stored in the blocks. The hash value was generated using a chaotic method. Each block contains the preceding block's hash value, timestamp, and hash value. In the form of a Blockchain, these blocks are connected. The institution enters student information into the suggested interface (application) by giving information such as name and email address, which is then kept in the database. The registrar's certificate is saved in the application, and they create a Blockchain. By entering the student's information, the employer or verifier can validate the certificate.

The input-write-validate-seal architecture presented in this study [8] improves and strengthens the trust and openness of existing educational administration systems in schools and universities. The proposed design is known as the four-phase style. Meanwhile, the current traditional design only includes two phases: input-write and outputwrite. These two phases for validating educational certificates using educational data, including assessments, student identity, and credential information, are input from desktop software or a web-based portal in both systems.

In paper [9] The certificate is stored in JSON and pdf format. The pdf copy is uploaded to the interplanetary file system node and the hash pointer is saved. The JSON file is 


\title{
International Journal of Engineering Technology and Management Sciences
}

\author{
Website: ijetms.in Issue: 1 Volume No.6 January - 2022 DOI: 10.46647/ijetms.2022.v06i01.002
}

ISSN: 2581-4621

imported by Hyperledger to create an asset. IPFS is a peerto-peer distributed file system that aims to link computing devices through a shared file system. A third-party verifier is one of the proposed participants. By using this system, the verifier can easily verify without any difficulties. The verifier receives a $\mathrm{QR}$ code from the student, using that the verifier can view the certificate. All the history of the student mark list is recorded. So, without any doubt, the verifier can easily understand.

In paper [10] analyses the system of students who will submit their details, email ID, government ID proofs, and biometric data. They will also submit a secret phrase. The biometric and the secret phrase will be hashed and stored on the Blockchain in the form of a transaction. The student will also retain a copy of this hash. The college will issue the digitally signed degree certificate to a student using its private key. This signed certificate will be encrypted and uploaded on the IPFS server. The hash of this encrypted, the signed certificate will be stored on the Blockchain. If a student wants to take admission to another college, he/she will submit the hash of his/her fingerprint and the secret phrase. The verifying college will retrieve the hash stored on the Blockchain. They will compare the hash of the student's fingerprint and the secret phrase with the hash stored on the Blockchain. If a match is found, the student's identity is valid. The student will also submit his/her previous degree certificates. Compute the hash of the submitted certificates and compare it with the hash stored on the Blockchain. If the hashes match, the certificates are valid and there is no degree of fraud.

In paper[11] analyses about financials to deploy two different business models may be used to establish a plan for higher education accreditation and degree verification. Both business models are based on a pay-per-certification and pay-per-certification model, with the pay-percertification model balancing the two. The certification and verification of degrees using Blockchain technology is a big market that could do well in the long run if it takes advantage of the commonalities that come from aggregating. Although aggregation decreases financial risk, it also increases the complexity of business development.

Credential forgery is indeed a chronic \& pervasive phenomenon that undermines faith in academic institutions, hinders societal growth, and costs a lot of money, according to a study [12]. Regrettably, inefficient, costly, and time-consuming credential verification solutions still exist. They are also ineffectual against some forms of systemic corruption, such as educational fraud. They've also introduced Cerberus, a full Blockchain-based verification system.
They describe a Blockchain-based architecture in paper [13], which improves the verification processes. As a result, the risk of certificate fraud is minimized, and graduation certificates' security, validity, and confidentiality are improved. Both issuing authorities and recipients, as well as consumers, will benefit from the proposed paradigm.

In paper [14] Education providers will be able to give the formal certificates providing proof of the completion using this technology, which incorporates powerful Blockchain technologies. Individuals can acquire these certificates and distribute them to people who need the official documents of the individual. And, education is a lifelong undertaking, not just a one-time event. People continue to learn because of their life experiences, professional training, and employment. It's just as important to keep track of those learning experiences as it is to keep track of official education. Our resolve can also provide after-college education with the necessary features.

In paper [15] implemented the verification procedure was proposed as a Blockchain approach for the verification of graduate certificates. As a result, the occurrence of certificate falsifications will be minimized, while certificate secrecy, security, and validity will be improved. This paper offers various benefits to approving authorities, receivers, and customers[16-50]. The Blockchain itself provides all of the information required to check and validate the certificate, which is an advantage of this paper. To validate the given certificates, the organization need not have contact with the institution/university[51-100.

\section{PROPOSED SYSTEM}

False certifications have long been a concern at academic organizations. Blockchain technology has lately emerged as a viable means of verifying the document verification process, as well as a powerful tool for combating document fraud and misuse. This approach will assist educational institutions in verifying a specific individual's certificates in a very short amount of time, with ease, and at a low cost. The primary goal of this system is to enable certificate verification and authentication[17]. The proposed system uses the application that is used to view, upload and verify the certificates, the application is built on the Blockchain network.

\section{A. Enhanced Security}

Our data is sensitive and important, and Blockchain can change the way we think about it. By creating a record that can't be changed and is encrypted end-to-end, Blockchain helps to prevent fraud and illegal behavior. Privacy problems on Blockchain can be addressed by anonymizing 


\title{
International Journal of Engineering Technology and Management Sciences
}

\author{
Website: ijetms.in Issue: 1 Volume No.6 January - 2022 DOI: 10.46647/ijetms.2022.v06i01.002
}

ISSN: $2581-4621$

personal data and limiting access via permissions. Hackers have a difficult time obtaining data since it is stored over a network of computers rather than on a single server. The hash function is used by the application to verify the certificates created when they are uploaded to the Blockchain network.

\section{B. Greater transparency}

Each organization would have to maintain its database if Blockchain didn't exist. Because Blockchain uses a distributed ledger, transactions and data are recorded in the same way in multiple places[18]. All network users with permissioned access see the same information at the same time, ensuring perfect openness. All transactions are timeand date-stamped and are logged irreversibly. Members can now view the entire transaction history, effectively eliminating the chance of fraud. All activities of logs of transactions performed into the Blockchain network may be seen by the admin or AICTE who runs the application (more details in subsequent sections).

\section{Instant traceability}

Blockchain provides an audit trail that documents the origins of an asset at each stage of its journey. This aids in the verification of products in countries where consumers are concerned about environmental or human rights issues, or in enterprises plagued by counterfeiting and fraud. With Blockchain, it is possible to immediately share data about provenance with customers. Traceability data can also expose faults in any supply chain, such as when products are waiting to be shipped on a loading dock. Even if fraud occurs, because the transaction records are publicly viewable, tracing fraud and catching the perpetrators is simple. The application's and network's traceability is excellent.

\section{Increased efficiency and speed}

Traditional paper-based processes are inefficient, prone to human error, and frequently necessitate the involvement of a third party. By leveraging Blockchain to streamline these operations, transactions may be completed faster and more efficiently. The Blockchain can store both documentation and transaction data, eliminating the need for paper exchange. Because several ledgers do not need to be reconciled, clearing and settlement can be performed much faster. The proposed application is extremely quick due to the high efficiency of the technologies used.

\section{E. Automation}

The proposed application has smart contracts (shown in fig. 2) Smart contracts may potentially automate transactions, boosting your productivity and speeding up the process even further. After pre-specified requirements are met, the next stage of the transaction or process is initiated automatically. Smart contracts do away with the need for human intervention and rely on third parties to ensure that contractual obligations are met. When a consumer submits the required documentation for a claim, for example, the claim can be settled and compensated right away.

\section{F. Confidentiality, Integrity, and Availability}

Transactions are collected into blocks in a standard Blockchain, which is then preserved in a chain of blocks that are connected, with having each new block linked chronologically to the already generated hash of the previous block. The data elements in a Blockchain are not stored in a single location. Instead, data elements are stored across the Blockchain network, ensuring that the data components saved in the Blockchain remain secure. The principles of confidentiality, integrity, and availability are enforced by conventional information security practice. The secrecy components of Blockchain implementation are not enforced as strictly as the integrity and availability of the data contained inside it.

The data elements are transparent to all persons who share their data components with others on a single Blockchain due to its decentralized nature. As a result, the technology's secrecy component is difficult to enforce. All participants in a Blockchain can view the data that is sent into it, and there are no constraints on who may see it. Because of its decentralized nature, Blockchain has had unintended implications, such as allowing anybody to see and write transactions to a Blockchain. As a result, Bitcoin transactions have driven a large amount of illicit market trade activity throughout the world. Because the data stored on the Blockchain is not modifiable at this moment, the integrity of data pieces can be readily ensured[19]. However, it is worth noting that sufficient research is being conducted on enhancing the network architecture and providing a suitable level of secrecy, integrity, and privacy of transactions recorded in Blockchain.

\section{G. Working Flow Diagram}

The proposed system comes with the application that is used by the AICTE or admin, verifiers, and users/organization. Admin is the person who controls the application by taking charge of the person who can verify the certificates and he/she can only be the person who can upload the verified certificates into the Blockchain. Admin creates verifiers for each academic prospect, those verifiers are the persons whose details like name, the password for the verifier to access, which type like SSC or inter-board or degree or Ph.D./PG, contact number, email ID, home address are taken and then given them access i.e., username $\&$ password to access the application to upload the verified certificates to the application, which are thoroughly verified by the assigned verifiers and then the verified 


\section{International Journal of Engineering Technology and Management Sciences}

Website: ijetms.in Issue: 1 Volume No.6 January - 2022 DOI: 10.46647/ijetms.2022.v06i01.002

ISSN: $2581-4621$

certificates are in the application ready for the admin to upload into the Blockchain.

Once the verified certificates are uploaded into the Blockchain the hash code is generated and those are uneditable. The users/organization can be used to view the verified certificates in the application of the respective person. In the below figure, the Blockchain network can have one or more educational boards, the architecture of the project contains a mem pool that contains all the certificate records. Those records are given to the verifier, the verifier is SSC, intermediate, degree, $\mathrm{PG}$, and $\mathrm{Ph} . \mathrm{D}$. When the SSC certificate comes to verify then it is given to the SSC verifier and the SSC verifier can only verify the SSC certificate and all the remaining certificates are kept view only, this process applies to all the other verifiers also. Once the certificates are completely verified by all the verifiers then only the AICTE or admin can access them in the application and upload them in the Blockchain ledger.

Each person has a block with a unique ID to access them. Once the block is created it cannot tamper. User/Organization will have to get the permit to view or access the person's certificates.

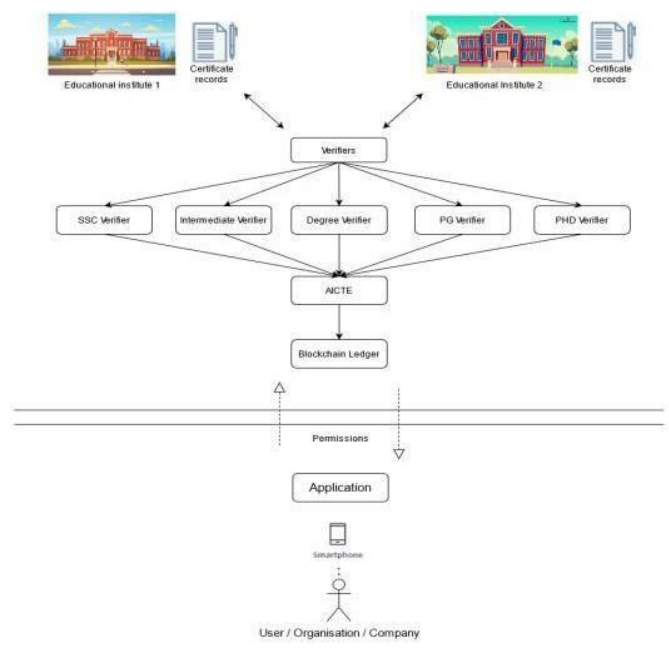

Fig. 1. Proposed System Architecture

\section{IMPLEMENTATION}

In this project, we have developed a web-based application that is using Blockchain technology to store academic certificates to avoid certificate counterfeit as lots of fake certificates are using to get jobs and there is no current technology to detect such fake certificates and to prevent such forgery, we are using Blockchain-based certificate verification. This web-based application consists of the following modules

1) Admin or AICTE Module: Admin can log in to the system by using the username as 'admin' and password as 'admin' and then admin will perform the following actions

a) Add Verification Officials: Using this module admin can add various verifier officials such as SSC verifier, Intermediate, Ph.D., and PG.

b) Upload Verified Certificates to Blockchain: using this module admin will upload verified certificates to Blockchain. For each certificate image, one hash code will be generated, and this hash code will be stored at Blockchain memory. Whenever we upload any test certificate then Blockchain verifies test certificate hash code with stored hash code and if match found then certificated will be authenticated and certificate owner details will be fetched and display otherwise certificate authentication will be failed.

c) Search Verified Certificates: In this module admin will upload the test certificate and then the application generates hash code from the certificate and matched it with Blockchain store images and if a match is found then the certificate will be authenticated.

2) Verify Officials Login: Using this module officials will $\log$ in to the application by using the username and password given by the admin user and then will upload and view certificates.

The web-based application which is developed to run the proposed framework is run on the tomcat server and Ethereum is used to implement the Blockchain. Sha hash function is used in the Blockchain and deployed the code using solidity and Ethereum. It will give the default 5000 gas value, as it trails for the academic purpose so it will use minimum gas value.

When the admin uploads the certificates that are provided by the verifier to the Blockchain then a new hash code is generated, and that certificate is uploaded into the Blockchain. The below fig. 2 shows the web application where the certificate has a hash code in the hash code column which means that a particular person's certificate is uploaded into the Blockchain 


\section{International Journal of Engineering Technology and Management Sciences}

Website: ijetms.in Issue: 1 Volume No.6 January - 2022 DOI: 10.46647/ijetms.2022.v06i01.002

ISSN: $2581-4621$

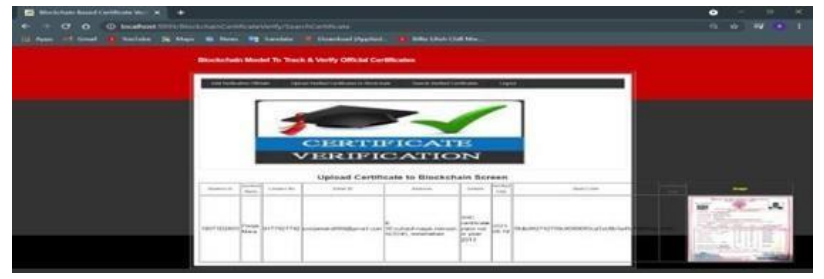

Fig. 2. Successfully verified certificate with hash code generated

The fig. 3 below shows when the fake certificate is uploaded into the blockchain then the blockchain denies and shows the error message of verification failed in the web application. When the verification failed message is shown it means the given certificate is fake.

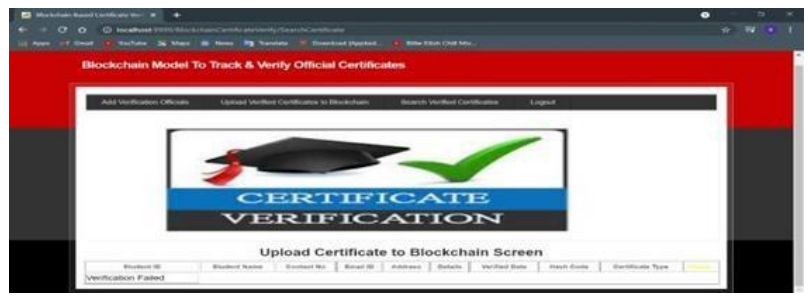

Fig. 3. When the fake certificate is uploaded verification fails.

\section{CONCLUSION}

This proposed system is developed with Blockchain hence utmost authentication is provided for the educational certificates that can be verified and stored, when the fake certificates arise then it can diminish, which will help the hard-earned people to get jobs in their desired organizations and all the fake circulation of certifications is stopped because the system identifies the genuine certificates. The system will maintain a common platform for storing and accessing the documents to track the documents easily for the organizations to access the true documents.

\section{References}

[1] Satoshi Nakamoto, Bitcoin: A Peer-to-Peer Electronic Cash System, www.bitcoin.org.

[2] Shanmugh Priya R, Swetha. N: Online Certificate Validation Using Blockchain, Special Issue Published in Int. Jnl. Of Advanced Networking \& Applications (IJANA), March 302019.

[3] Omar S. Saleh, Osman Ghazali, Muhammad Ehsan Rana: a blockchain-based framework for educational certificates verification, Article in journal of critical reviews, 2019.

[4] Potluri T., Jahnavi S., Motupalli R. (2021) Mobilenet V2FCD: Fake Currency Note Detection. In: Luhach A.K., Jat D.S., Bin Ghazali K.H., Gao XZ., Lingras P. (eds) Advanced Informatics for Computing Research. ICAICR 2020.
Communications in Computer and Information Science, vol 1393. Springer, Singapore. https://doi.org/10.1007/978-981$\underline{16-3660-8 \_26}$

[5] M.Ravikanth, Madhavi Latha Navuluri "Enabling smart cities through the framework of IOT" in International journal Research and analytical reviews, Volume 6, Issue 1, January, 2019, EISSN:2348-1269 P-ISSN:2349-5158.

[6] Jayesh G. Dongre, Dr.Kishore.T.Patil, Sonali.M.Tikam, Vasudha.B.Gharath: Education Degree Fraud Detection and Student Certificate Verification using Blockchain,published in www.ijert.org, 13july, 2020.

[7] Suresh C., Ravikanth M., Srivani B., Satish T. (2021) Cognitive IoTBased Smart Fitness Diagnosis and Recommendation System Using a Three-Dimensional CNN with Hierarchical Particle Swarm Optimization. In: Gupta D., Hugo C. de Albuquerque V., Khanna A., Mehta P.L. (eds) Smart Sensors for Industrial Internet of Things. Internet of Things (Technology, Communications and Computing). Springer, Cham. https://doi.org/10.1007/978-3-030-52624-5_10.

[8] A. Gayathri, J.Jayachitra, Dr.S.Matilda: Certificate validation using blockchain, IEEE in ICSSS, 2020

[9] Ravi Kanth Motupalli, Dr. O. Naga Raju "Modelling Disaggregated Smart Home Bigdata for Behaviour Analytics of a Human using Distributed architectures" in journal of critical reviews, Volume 7, Issue 19, 2020, ISSN: 2394-5125, Page no: 522-530.

[10] Mili Rafi, sherin meri shaji, prof.Ashley Thomas: certificate management and validation system using blockchain in IRJET.

[11] Ravi Kanth Motupalli, Kota Chaitanya Kumar "Arduino Uno based Smart Helmet with Protective Bike System Using IoT" in Journal of Advanced Research in Dynamical \& Control Systems, Vol. 12, No. 9, 2020, ISSN 1943-023X, Page no: 27-33.

[12] Prof.Jignasha Dala, meenal Chaturvedi, Himani gander, Sanjana thumb are verification of identity and educational certificates of students using biometric and blockchain in SSRN in 2020.

[13] Oliver, Miquel; Moreno, Joan; Prieto, Gerson; Benítez, David: Using blockchain as a tool for tracking and verification of official degrees: a business model in ZBW in 2020.

[14] Ravi Kanth Motupalli, Dr. O. Naga Raju "A Systematic Review on Modelling of Human Activity Patterns in Smart Homes" in Science, Technology and Development, Volume VIII Issue XII DECEMBER 2019, ISSN: 0950-0707, Page no: 942-950.

[15] Aamna Tariq, Hina Binte Haq, Syed Taha Ali: Cerberus: A Blockchain-Based Accreditation and Degree Verification System in SEECS 2020.

[16] Ravi Kanth Motupalli, Dr. O. Naga Raju” Integration of SQL Modelling and Graph Representations to Disaggregated Human Activity Data for Effective Knowledge Extraction" in PSYCHOLOGY AND EDUCATION (2020) 57(8): 975-984, ISSN: 00333077 .

[17] Meng Han, Zhigang Li, Dalei Wu, Ying Xie, Asif Baba: A Novel Blockchain-based Education Records Verification Solution in SIGITE 2020.

[18] Thin Zar Ko-Ko: Certificate Verification System Based on Blockchain Technology in University journal of creative and innovative research 2020 .

[19] Ravi Kanth Motupalli, Dr. Joel Sunny Deol Gosu, Dr. Pullagura Priyadarsini, "A Hybrid Approach for the Analysis of Feature Selection using Information Gain and BAT Techniques on The Anomaly Detection" in Turkish Journal of Computer and Mathematics Education, Vol.12 No. 5 (5th April ,2021), 656-666. 


\title{
International Journal of Engineering Technology and Management Sciences
}

\author{
Website: ijetms.in Issue: 1 Volume No.6 January - 2022 DOI: 10.46647/ijetms.2022.v06i01.002
}

ISSN: 2581-4621

[20] Sahoo, K. S., Tripathy, B. K., Naik, K., Ramasubbareddy, S., Balusamy, B., Khari, M., \& Burgos, D. (2020). An evolutionary SVM model for DDOS attack detection in software defined networks. IEEE Access, 8, 132502-132513.

[21] Vaishali, R., Sasikala, R., Ramasubbareddy, S., Remya, S., \& Nalluri, S. (2017, October). Genetic algorithm based feature selection and MOE Fuzzy classification algorithm on Pima Indians Diabetes dataset. In 2017 international conference on computing networking and informatics (ICCNI) (pp. 1-5). IEEE.

[22] Mohanta, B. K., Jena, D., Ramasubbareddy, S., Daneshmand, M., \& Gandomi, A. H. (2020). Addressing security and privacy issues of IoT using blockchain technology. IEEE Internet of Things Journal, 8(2), 881-888.

[23] Reddy, S. R. S., Nalluri, S., Kunisetti, S., Ashok, S., \& Venkatesh, B. (2019). Content-based movie recommendation system using genre correlation. In Smart Intelligent Computing and Applications (pp. 391-397). Springer, Singapore.

[24] Basu, S., Kannayaram, G., Ramasubbareddy, S., \& Venkatasubbaiah, C. (2019). Improved genetic algorithm for monitoring of virtual machines in cloud environment. In Smart Intelligent Computing and Applications (pp. 319-326). Springer, Singapore.

[25] Somula, R. S., \& Sasikala, R. (2018). A survey on mobile cloud computing: mobile computing+ cloud computing $(\mathrm{MCC}=\mathrm{MC}+$ CC). Scalable Computing: Practice and Experience, 19(4), 309-337.

[26] Sahoo, K. S., Mishra, P., Tiwary, M., Ramasubbareddy, S., Balusamy, B., \& Gandomi, A. H. (2019). Improving end-users utility in software-defined wide area network systems. IEEE Transactions on Network and Service Management, 17(2), 696-707.

[27] Sankar, S., Srinivasan, P., Luhach, A. K., Somula, R., \& Chilamkurti, N. (2020). Energy-aware grid-based data aggregation scheme in routing protocol for agricultural internet of things. Sustainable Computing: Informatics and Systems, 28, 100422 .

[28] Nalluri, S., Saraswathi, R. V., Ramasubbareddy, S., Govinda, K., \& Swetha, E. (2020). Chronic heart disease prediction using data mining techniques. In Data engineering and communication technology (pp. 903-912). Springer, Singapore.

[29] Somula, R., Anilkumar, C., Venkatesh, B., Karrothu, A., Kumar, C. P., \& Sasikala, R. (2019). Cloudlet services for healthcare applications in mobile cloud computing. In Proceedings of the 2nd International Conference on Data Engineering and Communication Technology (pp. 535-543). Springer, Singapore.

[30] Sai, K. B. K., Subbareddy, S. R., \& Luhach, A. K. (2019). IOT based air quality monitoring system using MQ135 and MQ7 with machine learning analysis. Scalable Computing: Practice and Experience, 20(4), 599-606.

[31] Sennan, S., Balasubramaniyam, S., Luhach, A. K., Ramasubbareddy, S., Chilamkurti, N., \& Nam, Y. (2019). Energy and delay aware data aggregation in routing protocol for Internet of Things. Sensors, 19(24), 5486.

[32] Somula, R., \& Sasikala, R. (2018). Round robin with load degree: An algorithm for optimal cloudlet discovery in mobile cloud computing. Scalable Computing: Practice and Experience, 19(1), 3952 .

[33] Sennan, S., Somula, R., Luhach, A. K., Deverajan, G. G., Alnumay, W., Jhanjhi, N. Z., ... \& Sharma, P. (2020). Energy efficient optimal parent selection based routing protocol for Internet of Things using firefly optimization algorithm. Transactions on Emerging Telecommunications Technologies, e4171.
[34] Somula, R., \& Sasikala, R. (2019). A load and distance aware cloudlet selection strategy in multi-cloudlet environment. International Journal of Grid and High Performance Computing (IJGHPC), 11(2), 85-102.

[35] Sankar, S., Ramasubbareddy, S., Luhach, A. K., Nayyar, A., \& Qureshi, B. (2020). CT-RPL: Cluster tree based routing protocol to maximize the lifetime of Internet of Things. Sensors, 20(20), 5858.

[36] Baliarsingh, S. K., Vipsita, S., Gandomi, A. H., Panda, A., Bakshi, S., \& Ramasubbareddy, S. (2020). Analysis of high-dimensional genomic data using MapReduce based probabilistic neural network. Computer methods and programs in biomedicine, 195, 105625 .

[37] Lin, S. Y., Du, Y., Ko, P. C., Wu, T. J., Ho, P. T., \& Sivakumar, V. (2020). Fog Computing Based Hybrid Deep Learning Framework in effective inspection system for smart manufacturing. Computer Communications, 160, 636-642.

[38] Somula, R., \& Sasikala, R. (2019). A honey bee inspired cloudlet selection for resource allocation. In Smart Intelligent Computing and Applications (pp. 335-343). Springer, Singapore.

[39] Sennan, S., Ramasubbareddy, S., Balasubramaniyam, S., Nayyar, A., Abouhawwash, M., \& Hikal, N. A. (2021). T2FL-PSO: Type-2 Fuzzy Logic-based Particle Swarm Optimization Algorithm used to Maximize the Lifetime of Internet of Things. IEEE Access, 9, 63966-63979.

[40] Somula, R., \& Sasikala, R. (2019). A research review on energy consumption of different frameworks in mobile cloud computing. Innovations in Computer Science and Engineering, 129142.

[41] Sathish, K., Ramasubbareddy, S., \& Govinda, K. (2020). Detection and localization of multiple objects using VGGNet and single shot detection. In Emerging Research in Data Engineering Systems and Computer Communications (pp. 427-439). Springer, Singapore.

[42] Krishna, A. V., Ramasubbareddy, S., \& Govinda, K. (2020). Task scheduling based on hybrid algorithm for cloud computing. In International Conference on Intelligent Computing and Smart Communication 2019 (pp. 415-421). Springer, Singapore.

[43] Somula, R., Kumar, K. D., Aravindharamanan, S., \& Govinda, K. (2020). Twitter sentiment analysis based on US presidential election 2016. In Smart Intelligent Computing and Applications (pp. 363373). Springer, Singapore.

[44] Sahoo, K. S., Tiwary, M., Sahoo, B., Mishra, B. K., RamaSubbaReddy, S., \& Luhach, A. K. (2019). RTSM: response time optimisation during switch migration in software-defined wide area network. IET Wireless Sensor Systems, 10(3), 105-111.

[45] Nalluri, S., Ramasubbareddy, S., \& Kannayaram, G. (2019). Weather prediction using clustering strategies in machine learning. Journal of Computational and Theoretical Nanoscience, 16(5-6), 1977-1981.

[46] Prasanna, S., Narayan, S., NallaKaruppan, M. K., Anilkumar, C., \& Ramasubbareddy, S. (2019). Iterative Approach for Frequent Set Mining Using Hadoop Over Cloud Environment. In Smart Intelligent Computing and Applications (pp. 399-405). Springer, Singapore.

[47] Somula, R., Narayana, Y., Nalluri, S., Chunduru, A., \& Sree, K. V. (2019). POUPR: properly utilizing user-provided recourses for energy saving in mobile cloud computing. In Proceedings of the 2nd International Conference on Data Engineering and Communication Technology (pp. 585-595). Springer, Singapore.

[48] Saraswathi, R. V., Nalluri, S., Ramasubbareddy, S., Govinda, K., \& Swetha, E. (2020). Brilliant corp yield prediction utilizing internet of 


\title{
International Journal of Engineering Technology and Management Sciences
}

\author{
Website: ijetms.in Issue: 1 Volume No.6 January - 2022 DOI: 10.46647/ijetms.2022.v06i01.002
}

ISSN: 2581-4621

things. In Data Engineering and Communication Technology (pp. 893-902). Springer, Singapore.

[49] Sankar, S., Srinivasan, P., Ramasubbareddy, S., \& Balamurugan, B. (2020). Energy-aware multipath routing protocol for internet of things using network coding techniques. International Journal of Grid and Utility Computing, 11(6), 838-846.

[50] Ramasubbareddy, S., Srinivas, T., Govinda, K., \& Manivannan, S. S. (2020). Comparative study of clustering techniques in market segmentation. In Innovations in Computer Science and Engineering (pp. 117-125). Springer, Singapore.

[51] Devi, B. L., Bai, V. V., Ramasubbareddy, S., \& Govinda, K. (2020). Sentiment analysis on movie reviews. In Emerging Research in Data Engineering Systems and Computer Communications (pp. 321-328). Springer, Singapore.

[52] Kumar, I. P., Gopal, V. H., Ramasubbareddy, S., Nalluri, S., \& Govinda, K. (2020). Dominant color palette extraction by K-means clustering algorithm and reconstruction of image. In Data Engineering and Communication Technology (pp. 921-929). Springer, Singapore.

[53] Srinivas, T. A. S., Ramasubbareddy, S., Govinda, K., \& Manivannan, S. S. (2020). Web image authentication using embedding invisible watermarking. In International Conference on Intelligent Computing and Smart Communication 2019 (pp. 207218). Springer, Singapore

[54] Mohanta, B. K., Jena, D., Mohapatra, N., Ramasubbareddy, S., \& Rawal, B. S. (2021). Machine learning based accident prediction in secure iot enable transportation system. Journal of Intelligent \& Fuzzy Systems, (Preprint), 1-13.

[55] Ramasubbareddy, S., Aditya Sai Srinivas, T., Govinda, K., \& Manivannan, S. S. (2020). Crime prediction system. In Innovations in Computer Science and Engineering (pp. 127-134). Springer, Singapore.

[56] Krishna, A. V., Ramasubbareddy, S., \& Govinda, K. (2020). A unified platform for crisis mapping using web enabled crowdsourcing powered by knowledge management. In International Conference on Intelligent Computing and Smart Communication 2019 (pp. 195-205). Springer, Singapore.

[57] Ramasubbareddy, S., Ramasamy, S., Sahoo, K. S., Kumar, R. L., Pham, Q. V., \& Dao, N. N. (2020). Cavms: Application-aware cloudlet adaption and vm selection framework for multicloudlet environment. IEEE Systems Journal.

[58] Maddila, S., Ramasubbareddy, S., \& Govinda, K. (2020). Crime and Fraud detection using clustering techniques. Innovations in Computer Science and Engineering, 135-143.

[59] Das, S. R., Ray, P. K., Sahoo, A. K., Ramasubbareddy, S., Babu, T. S., Kumar, N. M., ... \& Siano, P. (2020). Performance of Hybrid Filter in a Microgrid Integrated Power System Network Using Wavelet Techniques. Applied Sciences, 10(19), 6792.

[60] Mahesh, B., Kumar, K. P., Ramasubbareddy, S., \& Swetha, E. (2020). A review on data deduplication techniques in cloud. In Embedded Systems and Artificial Intelligence (pp. 825-833). Springer, Singapore.

[61] Pradeepthi, C., Geetha, V. V., Ramasubbareddy, S., \& Govinda, K. (2020). Prediction of real estate price using clustering techniques. In Emerging Research in Data Engineering Systems and Computer Communications (pp. 281-289). Springer, Singapore.

[62] Srinivas, A. S. T., Somula, R., Govinda, K., Saxena, A., \& Reddy, P. A. (2020). Estimating rainfall using machine learning strategies based on weather radar data. International Journal of Communication Systems, 33(13).
[63] Ramasubbareddy, S., \& Sasikala, R. (2021). RTTSMCE: a response time aware task scheduling in multi-cloudlet environment. International Journal of Computers and Applications, 43(7), 691-696.

[64] Ravindranath, V., Ramasamy, S., Somula, R., Sahoo, K. S., \& Gandomi, A. H. (2020, July). Swarm intelligence based feature selection for intrusion and detection system in cloud infrastructure. In 2020 IEEE Congress on Evolutionary Computation (CEC) (pp. 16). IEEE.

[65] Rakshitha, K., Rao, A. S., Sagar, Y., \& Ramasubbareddy, S. (2020). Demonstrating broadcast aggregate keys for data sharing in cloud. Innovations in Computer Science and Engineering, 185-193.

[66] Lavanya, V., Ramasubbareddy, S., \& Govinda, K. (2020). Fuzzy keyword matching using $\mathrm{N}$-gram and cryptographic approach over encrypted data in cloud. In Embedded Systems and Artificial Intelligence (pp. 551-558). Springer, Singapore.

[67] Chandrika, G. N., Ramasubbareddy, S., Govinda, K., \& Swetha, E. (2020). Web scraping for unstructured data over web. In Embedded Systems and Artificial Intelligence (pp. 853-859). Springer, Singapore.

[68] Kumar, I. P., Sambangi, S., Somukoa, R., Nalluri, S., \& Govinda, K. (2020). Server security in cloud computing using block-chaining technique. In Data Engineering and Communication Technology (pp. 913-920). Springer, Singapore.

[69] Bhukya, K. A., Ramasubbareddy, S., Govinda, K., \& Srinivas, T. A. S. (2020). Adaptive mechanism for smart street lighting system. In Smart Intelligent Computing and Applications (pp. 69-76). Springer, Singapore.

[70] Rekha, G., D Sravanthi, B., Ramasubbareddy, S., \& Govinda, K. (2019). Prediction of stock market using neural network strategies. Journal of Computational and Theoretical Nanoscience, 16(5-6), 2333-2336.

[71] Somula, R., Nalluri, S., NallaKaruppan, M. K., Ashok, S., \& Kannayaram, G. (2019). Analysis of CPU scheduling algorithms for cloud computing. In Smart Intelligent Computing and Applications (pp. 375-382). Springer, Singapore.

[72] Basha, S. M., Rajput, D. S., Janet, J., Somula, R. S., \& Ram, S. (2020). Principles and Practices of Making Agriculture Sustainable: Crop Yield prediction using Random Forest. Scalable Computing: Practice and Experience, 21(4), 591-599.

[73] Sankar, S., Ramasubbareddy, S., Chen, F., \& Gandomi, A. H. (2020, December). Energy-Efficient Cluster-based Routing Protocol in Internet of Things Using Swarm Intelligence. In 2020 IEEE Symposium Series on Computational Intelligence (SSCI) (pp. 219224). IEEE.

[74] Chugani, S., Govinda, K., \& Ramasubbareddy, S. (2018, February). Data Analysis of Consumer Complaints in Banking Industry using Hybrid Clustering. In 2018 Second International Conference on Computing Methodologies and Communication (ICCMC) (pp. 7478). IEEE

[75] Panda, S. S., Jena, D., Mohanta, B. K., Ramasubbareddy, S., Daneshmand, M., \& Gandomi, A. H. (2021). Authentication and Key Management in Distributed IoT using Blockchain Technology. IEEE Internet of Things Journal.

[76] Samanta, S., Singhar, S. S., Gandomi, A. H., Ramasubbareddy, S., \& Sankar, S. (2020, September). A WiVi Based IoT Framework for Detection of Human Trafficking Victims Kept in Hideouts. In International Conference on Internet of Things (pp. 96-107). Springer, Cham.

[77] Revathi, A., Kalyani, D., Ramasubbareddy, S., \& Govinda, K. (2020). Critical review on course recommendation system with 


\title{
International Journal of Engineering Technology and Management Sciences
}

\author{
Website: ijetms.in Issue: 1 Volume No.6 January - 2022 DOI: 10.46647/ijetms.2022.v06i01.002
}

ISSN: $2581-4621$

various similarities. Embedded Systems and Artificial Intelligence, 843-852.

[78] Srinivas, T. A. S., Somula, R., \& Govinda, K. (2020). Privacy and security in Aadhaar. In Smart Intelligent Computing and Applications (pp. 405-410). Springer, Singapore.

[79] Ramasubbareddy, S., Vedavasu, G., Gopi Krishna, K. B., \& Savithri, A. (2019). PIOCM: Properly Identifying Optimized Cloudlet in Mobile Cloud Computing. Journal of Computational and Theoretical Nanoscience, 16(5-6), 1967-1971.

[80] Sravankumar, B., Anilkumar, C., Easwaramoorthy, S., Ramasubbareddy, S., \& Govinda, K. (2019). Iterative Sharpening of Digital Images. In Information Systems Design and Intelligent Applications (pp. 53-62). Springer, Singapore.

[81] Behera, A., Panigrahi, T. K., Pati, S. S., Ghatak, S., Ramasubbareddy, S., \& Gandomi, A. H. (2021). A hybrid evolutionary algorithm for stability analysis of 2-area multi-nonconventional system with communication delay and energy storage. International Journal of Electrical Power \& Energy Systems, 130, 106823.

[82] Jena, M. D., Singhar, S. S., Mohanta, B. K., \& Ramasubbareddy, S. (2021). Ensuring Data Privacy Using Machine Learning for Responsible Data Science. In Intelligent Data Engineering and Analytics (pp. 507-514). Springer, Singapore.

[83] Ramasubbareddy, S., Srinivas, T. A. S., Govinda, K., \& Swetha, E. (2021). Sales Analysis on Back Friday Using Machine Learning Techniques. In Intelligent System Design (pp. 313-319). Springer, Singapore.

[84] Attili, V. R., Annaluri, S. R., Gali, S. R., \& Somula, R. (2020). Behaviour and Emotions of Working Professionals Towards Online Learning Systems: Sentiment Analysis. International Journal of Gaming and Computer-Mediated Simulations (IJGCMS), 12(2), 2643.

[85] Vinodhini, V., Vishalakshi, A., Chandrika, G. N., Sankar, S., \& Ramasubbareddy, S. (2022). Predicting Vasovagal Syncope for Paraplegia Patients Using Average Weighted Ensemble Technique. Journal of Mobile Multimedia, 135-162.

[86] Sankar, S., Somula, R., Parvathala, B., Kolli, S., \& Pulipati, S. (2021). SOA-EACR: Seagull optimization algorithm based energy aware cluster routing protocol for wireless sensor networks in the livestock industry. Sustainable Computing: Informatics and Systems, 100645.

[87] Srilatha, P., Ramasubbareddy, S., \& Sudheer, D. (2021). Adaptive local neighborhood range based firefly algorithm for link prediction. International Journal of System Assurance Engineering and Management, 1-15.

[88] Rajput, D. S., Somula, R., \& Poluru, R. K. (2021). A Novel Architectural Model for Dynamic Updating and Verification of Data Storage in Cloud Environment. International Journal of Grid and High Performance Computing (IJGHPC), 13(4), 75-83.

[89] Rout, J. K., Dalmia, A., Rath, S. K., Mohanta, B. K., Ramasubbareddy, S., \& Gandomi, A. H. (2021). Detecting Product Review Spammers Using Principles of Big Data. IEEE Transactions on Engineering Management.

[90] Fu, H., Zhang, Y., Subbareddy, R., \& Vadivel, T. (2021). Mathematical modeling on workplace violence hazard assessment and security analysis using optimized grey dynamic system theory. Work, (Preprint), 1-9.

[91] Theetchenya, S., Ramasubbareddy, S., Sankar, S., \& Basha, S. M. (2021). Hybrid approach for content-based image retrieval. International Journal of Data Science, 6(1), 45-56.
[92] Vashishtha, M., Chouksey, P., Rajput, D. S., Reddy, S. R., Reddy, M. P. K., Reddy, G. T., \& Patel, H. (2021). Security and detection mechanism in IoT-based cloud computing using hybrid approach. International Journal of Internet Technology and Secured Transactions, 11(5-6), 436-451.

[93] Shukla, A. K., Pippal, S., Singh, D., \& Reddy, S. R. (2021). An evolutionary-based technique to characterise an anomaly in internet of things networks. International Journal of Internet Technology and Secured Transactions, 11(5-6), 452-469.

[94] Sundarakumar, M. R., Sankar, S., \& Reddy, S. R. S. (2021). An effective cloud-based smart home appliances automation in IoT using PHMM model. International Journal of Internet Technology and Secured Transactions, 11(5-6), 564-583.

[95] Sennan, S., Ramasubbareddy, S., Nayyar, A., Nam, Y., \& Abouhawwash, M. (2021). LOA-RPL: Novel Energy-Efficient Routing Protocol for the Internet of Things Using Lion Optimization Algorithm to Maximize Network Lifetime.

[96] Samanta, S., Panda, M., Ramasubbareddy, S., Sankar, S., \& Burgos, D. (2021). Spatial-Resolution Independent Object Detection Framework for Aerial Imagery. CMC-COMPUTERS MATERIALS \& CONTINUA, 68(2), 1937-1948.

[97] Sankar, S., Somula, R., Kumar, R. L., Srinivasan, P., \& Jayanthi, M. A. (2021). Trust-Aware Routing Framework for Internet of Things. International Journal of Knowledge and Systems Science (IJKSS), 12(1), 48-59.

[98] Mishra, B., Jena, D., Somula, R., \& Sankar, S. (2020). Secure Key Storage and Access Delegation Through Cloud Storage. International Journal of Knowledge and Systems Science (IJKSS), 11(4), 45-64.

[99] Palanisamy, S., Sankar, S., Somula, R., \& Deverajan, G. G. (2021) Communication trust and energy-aware routing protocol for WSN using DS theory. International Journal of Grid and HighPerformance Computing (IJGHPC), 13(4), 24-36.

[100] Ramasubbareddy, S., Walia, A. S., Luhach, A. K., Kannayaram, G., \& Evakattu, S. (2021). Big Data Analysis on Job Trends Using R. Recent Advances in Computer Science and Communications (Formerly: Recent Patents on Computer Science), 14(1), 100-108. 